\title{
Towards a Predictive Model of Power Loss from Parametric Decay of ICRF Waves
}

\author{
J. R. Myra, ${ }^{\dagger}$ L. A. Berry* and E. F. Jaeger* \\ † Lodestar Research Corporation, Boulder, Colorado \\ * Oak Ridge National Laboratory, Oak Ridge, Tennessee
}

May 2007

presented at the 17th Topical Conference on Radio Frequency Power in Plasmas May 7-9, 2007, Clearwater, Florida

\section{LODESTAR RESEARCH CORPORATION 2400 Central Avenue Boulder, Colorado 80301}




\title{
Towards a Predictive Model of Power Loss from Parametric Decay of ICRF Waves
}

\author{
J. R. Myra, ${ }^{\dagger}$ L. A. Berry* and E. F. Jaeger* \\ $\dagger$ Lodestar Research Corporation, Boulder, Colorado, USA \\ * Oak Ridge National Laboratory, Oak Ridge, Tennessee, USA
}

\begin{abstract}
Both theory and experiment have suggested that parametric decay instability (PDI) is a possible edge power loss mechanism in ICRF (including HHFW and IBW) heating of tokamak plasmas. In this paper, we consider the extension of previous theoretical models to enable a quantitative evaluation of pump depletion and power loss due to PDI. A set of nonlinear coupled equations for a long-wavelength "dipole" pump and short-wavelength daughter modes is derived. The model recovers the standard PDI dispersion relation for fixed pump wave amplitude, and obeys an appropriate nonlinear energy conservation law. When dissipation is present the model provides a description of energy flow from the pump to the daughter modes and the particles. The equations are intended to describe parametric decay and pump depletion in inhomogeneous plasmas. By writing the nonlinear coupling coefficients in terms of a spectral basis, a strategy for implementing the model in full-wave spectral codes is presented.
\end{abstract}

Keywords: parametric, decay, nonlinear, ICRF, full-wave, tokamak, edge

PACS: 52.35.Mw, 52.50.Qt, 52.55.Fa

\section{INTRODUCTION}

Parametric decay instability (PDI) represents one of several possible power loss channels for intense rf waves propagating through the tokamak edge plasma (from the antenna into the core). PDI has been observed on NSTX, ${ }^{1}$ C-MOD, ${ }^{2}$ ASDEX, ${ }^{3}$ DIII-D, ${ }^{4}$ TEXTOR, ${ }^{5}$ and JT-60. ${ }^{6}$ It is particularly virulent for direct IBW launch (because of the slow group velocity and hence large amplitude of IBW waves), but has also been observed in conventional (fast wave) ICRF experiments, and has recently been suggested as a possible explanation for observed power loss and concomitant edge ion heating during HHFW experiments on NTSX. ${ }^{1}$

Past theoretical work ${ }^{7}$ has clearly identified the possible PDI decay modes for ICRF heating of fusion plasmas, and established power thresholds for instability that are likely exceeded in some experiments. Here, we build on previous studies to construct a model set of coupled nonlinear equations that can be used to describe pump depletion (lost power). Strategies for implementation of this model in the fullwave spectral code AORSA ${ }^{8}$ are discussed. Our ultimate goal is a predictive numerical modeling capability for edge power loss due to PDI.

PDI is an inherently nonlinear process resulting from the coupling of the launched ("pump") wave into daughter modes. The usual frequency and wavenumber matching conditions for the three modes are described here by 


$$
\begin{aligned}
& \omega_{1}+\omega_{2}+\omega_{3}=0 \\
& \mathbf{k}_{1}+\mathbf{k}_{2}+\mathbf{k}_{3}=0
\end{aligned}
$$

where the mode conventions employed are: $1=$ pump; $2,3=$ daughters. The nonlinear beat currents, $\propto \mathbf{E}_{\mathrm{n}}^{*} \mathbf{E}_{\mathrm{m}}^{*}$ act as a source which drives the electric field $\mathbf{E}_{\mathrm{j}}$ where $(\mathrm{j}, \mathrm{n}, \mathrm{m})$ are a permutation of $(1,2,3)$. In this paper, we consider the so-called "dipole" limit for the pump wave

$$
\mathbf{k}_{1} \ll \mathbf{k}_{2}, \mathbf{k}_{3}
$$

so that $\mathbf{k}_{3} \approx-\mathbf{k}_{2}$.

Two types of decay processes can be considered: (i) resonant decay in which both daughter modes are propagating, i.e. the plasma dielectric $\varepsilon_{2} \equiv \varepsilon\left(\omega_{2}, \mathbf{k}_{2}\right)=0=\varepsilon_{3}$; and (ii) quasi-mode decay in which only one daughter mode (the lower sideband) is propagating, here taken to be mode 2, i.e. $\varepsilon_{2}=0$, while mode 3 (the lowest frequency decay product) is a quasi-mode, i.e. a wave-particle resonance, such as occurs near the ion cyclotron frequency $\omega_{3} \approx \Omega_{\mathrm{i}}$ or electron Landau resonance. Here, although most results are general, we concentrate on the quasi-mode case, for which Eqs. (1) and (2) are more easily satisfied.

\section{DERIVATION OF THE MODEL EQUATIONS}

Equations for the daughter modes are derived using the usual oscillating frame approach. (See e.g. Refs. 1, 7 and refs. therein.) The Vlasov equation can be written in terms of the velocity variable $\mathbf{w}=\mathbf{v}-\mathbf{u}(\mathrm{t})$ where $\mathbf{u}$ is the jitter velocity of particles in the spatially constant pump electric field. Coupling between modes arises from the term $\mathbf{u} \cdot \nabla f$. Here $\mathbf{u}$ is given by

$$
\mathbf{u}=\mathrm{M}_{1} \cdot \mathbf{a}_{1}
$$

where $\mathbf{a}_{1}=\mathrm{Ze} \mathbf{E}_{1} / \mathrm{m}$ and $\mathrm{M}_{1}$ is proportional to the cold-plasma dielectric tensor at the pump frequency $\omega_{1}$. Throughout the paper, subscripts denote mode frequency.

This oscillating frame calculation yields the perturbed (daughter wave) plasma density responses, e.g. $\mathrm{n}_{3}^{*} \propto \mathbf{E}_{1} \mathbf{E}_{2}, \mathrm{n}_{2} \propto \mathbf{E}_{1}^{*} \mathbf{E}_{3}^{*}$. To obtain the back-reaction of the daughter modes on the pump in the dipole limit, we consider the Vlasov equation

$$
\frac{\partial \mathrm{f}_{1}}{\partial \mathrm{t}}+\Omega \mathbf{v} \times \mathbf{b} \cdot \nabla_{\mathrm{v}} \mathrm{f}_{1}=-\mathbf{a}_{2}^{*} \cdot \nabla_{\mathrm{v}} \mathrm{f}_{3}^{*}-\mathbf{a}_{3}^{*} \cdot \nabla_{\mathrm{v}} \mathrm{f}_{2}^{*}
$$

Taking the velocity moment, the operator on the LHS will be recognized as the jitter operator from cold-fluid theory. Thus, employing Eq. (4) we have

$$
\mathbf{J}_{1}^{*}=\mathrm{ZeM}_{1}^{*} \cdot\left(\mathbf{a}_{2} \mathrm{n}_{3}+\mathbf{a}_{3} \mathrm{n}_{2}\right)
$$

Thus, is only necessary to substitute in the results for $n_{2}$ and $n_{3}$ from the oscillating frame calculation to obtain the beat-current at the pump frequency.

After considerable algebra, we obtain the following coupled nonlinear equations for the case of a dipole electromagnetic pump, and electrostatic daughters

$$
\begin{gathered}
\overrightarrow{\mathrm{L}}_{1}^{*} \cdot \mathbf{E}_{1}^{*}+\left(\overrightarrow{\mathrm{T}}_{12}^{*}\left|\mathrm{E}_{2}\right|^{2}+\overrightarrow{\mathrm{T}}_{13}^{*}\left|\mathrm{E}_{3}\right|^{2}\right) \cdot \mathbf{E}_{1}^{*}=\mathrm{C}_{23}: \mathbf{E}_{2} \mathbf{E}_{3} \\
\left(\overrightarrow{\mathrm{L}}_{2}+\overrightarrow{\mathrm{T}}_{2}\left|\mathrm{E}_{1}\right|^{2}\right) \cdot \mathbf{E}_{2}=\mathrm{C}_{13}^{*}: \mathbf{E}_{1}^{*} \mathbf{E}_{3}^{*}
\end{gathered}
$$




$$
\left(\overrightarrow{\mathrm{L}}_{3}+\overrightarrow{\mathrm{T}}_{3}\left|\mathrm{E}_{1}\right|^{2}\right) \cdot \mathbf{E}_{3}=\mathrm{C}_{12}^{*}: \mathbf{E}_{1}^{*} \mathbf{E}_{2}^{*}
$$

where $\overrightarrow{\mathrm{L}}$ is the usual wave operator $\overrightarrow{\mathrm{L}}_{\mathrm{j}} \cdot \mathbf{E}_{\mathrm{j}}=-\mathrm{c}^{2} / \omega_{\mathrm{j}}^{2} \nabla \times \nabla \times \mathbf{E}_{\mathrm{j}}+\vec{\varepsilon}_{\mathrm{j}} \cdot \mathbf{E}_{\mathrm{j}}$.

The coupling coefficients are given in terms of the quantities $\mathrm{z}=\mathbf{k}_{2} \cdot \mathbf{u}_{1} / \omega_{1}, \mathbf{M}_{1}$, and the linear species susceptibilities $\vec{\chi}_{2}, \vec{\chi}_{3}$. For example

$$
\mathrm{C}_{23}: \mathbf{E}_{2} \mathbf{E}_{3}=\sum \mathbf{k}_{2} \cdot \overrightarrow{\mathrm{M}}_{1} \frac{\mathrm{Ze}}{\mathrm{m} \omega_{1}}\left(\vec{\chi}_{3}-\vec{\chi}_{2}\right): \mathbf{E}_{2} \mathbf{E}_{3}
$$

where the sum is over species, and $\vec{\chi}_{j}=\vec{\chi}_{j}\left(\mathbf{k}_{2}\right)=\vec{\chi}_{j}\left(-\mathbf{k}_{3}\right)$.

\section{MODEL PROPERTIES AND NUMERICAL STRATEGY}

If we hold the pump fixed [i.e. ignore Eq. (7)] and consider the stability of infinitesimal daughters from Eqs. (8) and (9), we obtain the PDI dispersion relation

$$
\varepsilon_{2} \varepsilon_{3}^{*}=-\frac{1}{2} \sum_{\alpha, \beta}\left|\mathrm{z}_{\alpha}-\mathrm{z}_{\beta}\right|^{2} \mathrm{Y}_{\alpha} \mathrm{Y}_{\beta}
$$

which agrees with previous results. ${ }^{7}$ Here $Y=\chi_{2}-\chi_{3}^{*}$ and $\alpha, \beta$ are species indices. More generally, allowing all three modes to interact, we can derive an energy conservation law for $\mathrm{dW} / \mathrm{dt}$ where $\mathrm{W}=\mathrm{W}_{1}+\mathrm{W}_{2}+\mathrm{W}_{3}$ is the total energy in all the modes. In the absence of dissipation, i.e. $\operatorname{Im} \chi_{\alpha j}=0$ for all $\alpha$ and $j=1,2,3$, it can be shown that $\mathrm{dW} / \mathrm{dt}=0$. In this limit, the expected symmetries of the coupling coefficients are obeyed and the nonlinear equations describe reversible energy exchange among the modes. ${ }^{11}$

The higher order coupling terms $\mathrm{T}_{\mathrm{j}}$, while relatively unimportant for resonant 3wave PDI, are crucial for dissipative quasi-mode PDI. Considering for simplicity the case where the jitter velocity of one species is dominant, $|z| \equiv\left|z_{\alpha}\right|>>\left|z_{\beta}\right|$ for some $\alpha$, and letting mode 3 be the quasi-mode, it can be shown that

$$
\frac{\mathrm{dW}}{\mathrm{dt}}=-\gamma\left|\frac{\omega_{3}}{\omega_{2}}\right| \mathrm{W}_{2}
$$

where $\mathrm{W}_{2} /\left|\omega_{2}\right|$ is the number of "quanta" in the sideband daughter mode, we assume the only damping is on the quasi-mode, and $\gamma$ is the PDI growth rate,

$$
\gamma\left|\frac{\partial \varepsilon_{2}}{\partial \omega_{2}}\right|=|z|^{2} \operatorname{Im} \frac{Y_{\mathrm{i}} \mathrm{Y}_{\mathrm{e}}}{\mathrm{Y}_{\mathrm{i}}+\mathrm{Y}_{\mathrm{e}}}
$$

The energy in the pump wave decays at a faster rate (since $\left|\omega_{1}\right|>\left|\omega_{3}\right|$ ) given by

$$
\frac{\mathrm{dW}_{1}}{\mathrm{dt}}=-\gamma\left|\frac{\omega_{1}}{\omega_{2}}\right| \mathrm{W}_{2}
$$

with the difference residing in $\mathrm{W}_{2}$, the energy contained in the propagating sideband.

To obtain quantitative information for analysis and prediction of experiments, the nonlinear equations must be solved numerically in a full-wave model. The full-wave spectral code, AORSA, ${ }^{8}$ may be modified for this purpose. We consider the spatial problem with the $\omega_{\mathrm{j}}$ fixed. Then the nonlinear beat current terms $\mathbf{J}_{\mathrm{nl}}$ in Eqs. (7) - (9) act like a volume source term, or internal "antenna” driving field equations which are iterated to convergence. At the $\mathrm{r}^{\text {th }}$ iteration step, we have heuristically 


$$
\mathrm{L} \mathbf{E}^{(\mathrm{r}+1)}=-\frac{4 \pi \mathrm{i}}{\omega} \mathbf{J}_{\mathrm{nl}}\left(\mathbf{E}^{(\mathrm{r})}\right)
$$

In practice, the PDI must also be "seeded" with a noise current source.

We must also accommodate plasma inhomogeneity and the Fourier spectral representation of modes employed in AORSA, viz.

$$
\mathbf{E}(\mathrm{x})=\sum_{\mathrm{k}} \mathbf{E}_{\mathrm{k}} \mathrm{e}^{\mathrm{ikx}} ; \quad \mathbf{J}(\mathrm{x})=\sum_{\mathrm{k}} \sigma(\mathrm{k}, \mathrm{x}) \cdot \mathbf{E}_{\mathrm{k}} \mathrm{e}^{\mathrm{ikx}}
$$

In an inhomogeneous plasma, the strict dipole limit is relaxed by treating the pump's spatial variation $\mathbf{E}_{1}(\mathrm{x})$ and that of the coupling coefficients analogously to that of the $\mathrm{x}$ dependence of the linear conductivity $\sigma$. For example, in Eq. (10) the dielectric tensors $\chi(\mathrm{k})$ are generalized in terms of the so-called $\mathrm{W}$-matrix, $\mathrm{W}\left(\mathrm{k}, \mathrm{k}^{\prime}\right)$ employed previously for the calculations of local wave energy deposition ${ }^{9}$ and driven flows. ${ }^{10}$ Similarly, the coefficients $\mathrm{C}_{12}$ and $\mathrm{C}_{13}$ become

$$
\mathrm{C}(\mathrm{k} ; \mathrm{x}) \rightarrow \mathrm{C}(\mathrm{k}, \mathrm{k} ; \mathrm{x})-\left.\mathrm{i} \frac{\partial}{\partial \mathrm{x}} \frac{\partial \mathrm{C}\left(\mathrm{k}, \mathrm{k}^{\prime} ; \mathrm{x}\right)}{\partial \mathrm{k}^{\prime}}\right|_{\mathrm{k}^{\prime}=\mathrm{k}}
$$

It is expected that this procedure will maintain energy conservation and allow a quantitative full-wave numerical description of the PDI-driven decay of the pump as it propagates through the edge plasma. Important inhomogeneous effects for the case of HHFW $\rightarrow$ IBW + IQM decay include the drop of the pump amplitude away from the antenna, detuning of the ion-cyclotron quasi-mode (IQM) resonance [since $\omega_{3} \neq \Omega_{\mathrm{i}}(\mathrm{x})$ everywhere], and the spatial variation of $\mathrm{Y}_{\mathrm{e}} \sim \operatorname{Im} \chi_{3 \mathrm{e}} \sim \omega_{3} / \mathrm{k}_{\|} \mathrm{V}_{\text {te }}(\mathrm{x})$ in the edge plasma.

\section{ACKNOWLEDGMENTS}

Research sponsored by the Laboratory Directed Research and Development Program of Oak Ridge National Laboratory, managed by UT-Battelle, LLC, for the U.S. Department of Energy under Contract No. DE-AC05-00OR22725; and, in part, by the US DOE through grant DE-FG02-97ER54392.

\section{REFERENCES}

1. J. R. Wilson et al., AIP Conf. Proc. 787, 66 (2005).

2. J. C. Rost, M. Porkolab and R.L. Boivin, Phys. Plasmas 9, 1262 (2002).

3. R. Van Nieuwenhove, G. Van Oost, J.-M. Noterdaeme et al., Nucl. Fusion 28, 1603 (1988).

4. R. I. Pinsker, C. C. Petty, M. J. Mayberry, M. Porkolab et al., Nucl. Fusion 33, 777 (1993).

5. G. Van Oost, R. Van Nieuwenhove, R. Koch et al., Fusion Eng. Design 12, 149 (1990).

6. T. Fujii, M. Saigusa, H. Kimura, M. Ono et al., Fusion Eng. Design 12, 139 (1990).

7. M. Porkolab, Fus. Eng. Design 12, 93 (1990); and refs. therein.

8. E.F. Jaeger, L.A. Berry, E. D’Azevedo et al., Phys. Plasmas 8, 1573 (2001).

9. E.F. Jaeger, L.A. Berry and D.B. Batchelor, Phys. Plasmas 7, 3319 (2000).

10. J.R. Myra, L.A. Berry, D.A. D’Ippolito and E.F. Jaeger, Phys. Plasmas 11, 1786 (2004).

11. R.C. Davidson, Methods in Nonlinear Plasma Theory (Academic, New York, 1972). 